\title{
BMJ Open Factors influencing obesogenic dietary intake in young children ( $0-6$ years): systematic review of qualitative evidence
}

\author{
Veena Mazarello Paes, ${ }^{1,2}$ Ken K Ong, ${ }^{3}$ Rajalakshmi Lakshman ${ }^{3}$
}

To cite: Mazarello Paes V, Ong KK, Lakshman R. Factors influencing obesogenic dietary intake in young children ( $0-6$ years): systematic review of qualitative evidence. $B M J$ Open 2015;5:e007396. doi:10.1136/bmjopen-2014007396

- Prepublication history and additional material is available. To view please visit the journal (http://dx.doi.org/ 10.1136/bmjopen-2014007396).

Received 9 December 2014 Revised 8 July 2015 Accepted 31 July 2015

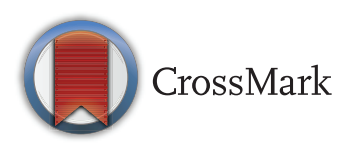

${ }^{1}$ Institute of Public Health, University of Cambridge, Cambridge, UK

${ }^{2}$ Institute of Child Health, University College London, London, UK

${ }^{3}$ MRC Epidemiology Unit \& UKCRC Centre for Diet and Activity Research (CEDAR), University of Cambridge, Cambridge, UK

Correspondence to Veena Mazarello Paes; veena.paes.14@ucl.ac.uk

\section{ABSTRACT}

Background: Obesogenic dietary intake is prevalent in young children and is associated with obesity and other adverse health outcomes in childhood and later in life.

Objective: To describe the barriers to and facilitators of obesogenic dietary intake in early childhood, in order to inform interventions and public health policies to prevent obesity.

Design: Systematic review of qualitative literature on factors influencing obesogenic diets in children aged 0 6 years.

Data sources: MEDLINE, EMBASE, CINAHL, PsycINFO, Web of Knowledge, British Nursing Index, ASSIA and Sociological Abstracts.

Review methods: Qualitative studies meeting the inclusion criteria were synthesised. Data were analysed by creating a thematic framework, underpinned by the socioecological model, which included familiarisation of data across the studies, indexing, charting, mapping and interpretation.

Results: 20 studies from the USA (10), Europe (6) and Australia (4) included the views of 1067 participants (901 parents/caregivers, 37 children, 87 teachers, 15 dieticians and 27 nursery staff). Study designs included focus groups $(n=16)$, individual interviews $(n=6)$ and ethnography $(n=1)$ with some studies using more than one design. Despite wide differences in the study context and focus, several consistent themes emerged. Parental factors increasing young children's obesogenic diets were: negative parent/family/peer modelling, lack of knowledge, time constraints, using food as reward, affordability and concerns about child's health. Child preferences also increased intake. Environmental factors increasing intake include: availability, advertising, societal, cultural and preschool/childcare influences.

Conclusions: Future intervention strategies should aim to promote modelling of positive behaviours, create home and preschool environments that promote healthy diets, and simultaneously target factors at the family and preschool/childcare levels.

Trial registration number: This review is one of a series of systematic reviews on the determinants of obesogenic behaviours in young children, registered with the International Prospective Register for Systematic Reviews (PROSPERO), CRD42012002881.

\section{Strengths and limitations of this study}

- To our knowledge, this is the first systematic review of qualitative evidence on factors influencing obesogenic dietary intake in preschool-aged children and we found that carers/parents, family and peer modelling significantly influenced young children's diet.

- We conducted a comprehensive search to identify relevant literature and followed strict systematic review procedures to minimize selection and reviewer related biases.

- Data was synthesized using a thematic framework, underpinned by the socio-ecological model.

- Ten (out of 12) factors/themes were consistently reported by nine or more studies.

- Almost all studies were conducted in developed countries and study participants were mainly mothers, which could limit the generalisability of the findings.

\section{INTRODUCTION}

Recent figures show alarming numbers of obese and overweight preschool-aged children. ${ }^{1}$ In 2010, 43 million children under the age of five were obese or overweight worldwide and the prevalence was estimated to rise from $6.7 \%$ to $9.1 \%$ in $2020 .{ }^{2}$ In 2013, around $22 \%$ of children in school aged $4-5$ years and around $33 \%$ children aged $10-11$ years were overweight or obese in England. ${ }^{3}$ In 2014, a study in the USA reported that almost half of the children who were obese in eighth grade were overweight or obese when they started kindergarten. ${ }^{4}$ Furthermore, they concluded that the child's weight status is set by age five and tracks throughout childhood. ${ }^{4}$ Childhood obesity is an increasing public health problem as it affects health and wellbeing adversely during childhood and adulthood. ${ }^{5}$ However, preventing child overweight and obesity remains an important and complex public health challenge. ${ }^{6-8}$ Evidence 
indicates that an individual's behaviour is influenced by a multitude of factors operating at different levels of the socioecological model of health behaviour. ${ }^{9} 10$

Quantitative evidence suggests that energy balance-related behaviours (EBRBs) such as consumption of energy dense, nutrient poor food (eg, unhealthy snacks) and drink (eg, sugar-sweetened beverages, SSBs) and sedentary behaviours (eg, TV viewing) are the main cause of the increase in overweight and obesity in children. ${ }^{11-13}$ Targeting these multiple EBRBs could help prevent overweight and obesity, since they are known to lie on the causal pathway of weight gain in young children. ${ }^{14-16}$ SSB consumption is associated with obesity, and predictive of excess weight gain ${ }^{17} 18$ and responsible for the higher caloric intakes among SSB consumers. ${ }^{19}$

There is increasing evidence that certain dietary intake patterns (obesogenic diet) are associated with obesity, excess weight gain and other adverse (physical, emotional, educational and social) outcomes in childhood and adulthood. ${ }^{20-23}$ For example, energy-dense diets that are poor in essential nutrients (non-core foods) have adverse effects on obesity prevalence as well as on general health status and well-being. ${ }^{6}$ Sedentary behaviours and obesogenic dietary intake patterns of fast food, snacks (high fat, sweet, salt) and SSB consumption, adopted during childhood, are known to track into adulthood. ${ }^{24-27}$ Consumption of SSBs (such as fruit juices/drinks or fruit-flavoured beverages which have high sugar content) in young children is a risk factor for overall poor diet ${ }^{19}$ and oral health. ${ }^{28} 29$

Early childhood is seen as a period of habit formation and a crucial time for interventions to prevent overweight and obesity. ${ }^{30}$ In order to change dietary behaviours, it is important to understand the factors influencing these behaviours and interventions targeting these factors may be more effective. ${ }^{14}{ }^{31}$ Modifying lifestyle has become an important avenue for investigation, since evidence suggests that complex interventions involving multiple methods are required to change dietary behaviour. ${ }^{30}$

However, most studies addressing this topic are quantitative and on school-aged children and adolescents, with few studies conducted specifically among preschool-aged children. Qualitative research informs quantitative research and guides the design of intervention and practice. ${ }^{32}$ This paper systematically synthesises the qualitative evidence on the factors influencing obesogenic dietary intake in preschool children underpinned by the socioecological model, in order to inform obesity preventative strategies.

\section{METHODS}

An iterative scoping stage (with input from experts) was followed by a combined search strategy with terms related to population (preschool children aged 06 years), exposure and outcome (fruit and vegetable consumption, SSB and other obesogenic diet consumption, physical activity and sedentary behaviours) to identify papers. The overall study design, search, inclusion/ exclusion and quality assessment strategies are previously described in the published protocol ${ }^{33}$ and follow review methods described by the Evidence for Policy and Practice Information (EPPI) Centre for the rigorous conduct and reporting of systematic reviews for policy and practice. ${ }^{34}$

\section{Search strategy}

The search strategy for literature was comprehensive and was without period or language restrictions (see online supplementary table S1). We also handsearched and contacted authors of included studies to identify relevant literature. A total of 37868 articles were identified through search of eight electronic databases in August 2012, after deduplication (MEDLINE $\mathrm{n}=20374$, EMBASE $n=17$ 331, CINAHL $n=775$, PsycINFO $n=1868$, Web of Knowledge $n=13455$, BNI $n=291$, ASSIA $n=113$ and Sociological Abstracts $n=135$ ). The flow chart of the literature search is presented in figure 1. A rerun search of June 2014 yielded 8908 articles after deduplication. The results of the 10 additional qualitative studies that met our inclusion criteria have been reported in the discussion. No additional themes emerged from these recent studies and the overall conclusions of our review did not change.

\section{Study selection}

Qualitative studies providing a greater understanding of the factors influencing obesogenic dietary intake in preschool-aged children were included (see inclusion/ exclusion criteria in online supplementary table S2). The following foods were included as obesogenic diets: snacks high in sugar, salt or fat (eg, crisps, chips, cakes, pastry, sweets and chocolate), combinations of foods (eg, fast food, junk food, convenience food and takeaway food), diets categorised by other researchers as obesogenic (eg, non-core foods, non-prudent diet and western diet) and SSBs (soft drinks, fruit juices, other sweetened drinks).

\section{Quality assessment}

A standard quality assessment tool for qualitative study designs, as specified by the EPPI centre, was used ${ }^{34}$ and investigated the following preset criteria: research questions clearly stated, approach appropriate for the research question, qualitative approach clearly justified, study context clearly described, role of the researcher clearly described, sampling method clearly described, sampling strategy appropriate for the research question, method of data collection clearly described, data collection method appropriate, method of analysis clearly described, analysis appropriate for the research question, and conclusions supported by sufficient evidence. Quality of the studies was based on the total quality assessment score-'high': met 9-12 quality criteria, 'intermediate': met 5-8 quality criteria, 'low': met 4 or less quality criteria. 
Figure 1 Flow chart of the systematic literature search for qualitative studies on obesogenic dietary intake in young children.
Total yield of papers on diet and physical activity from electronic database search (after de-duplication): $n=46,876$

Papers excluded during title \& abstract screen stage: $n=46,580$

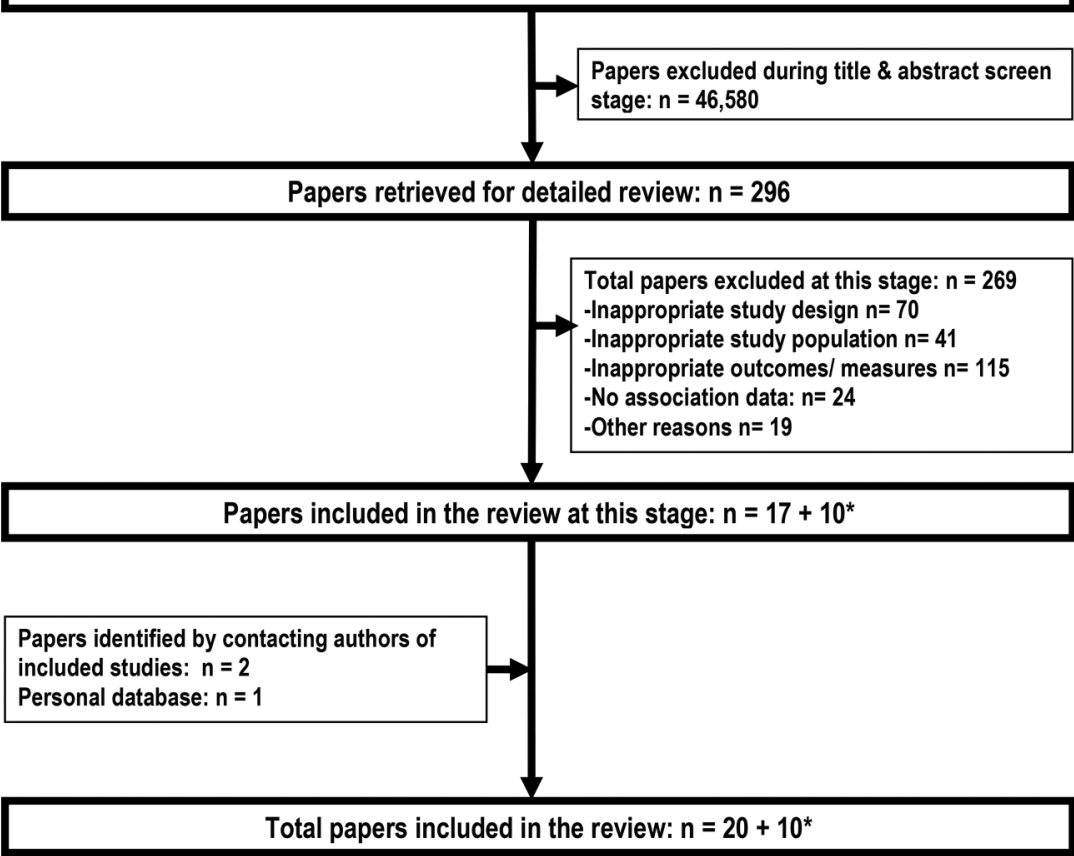

* Results of studies included only in discussion following an update to the search in June 2014.
Study selection and quality appraisal was performed by one author (VMP) and all included studies were double reviewed (RL). Of the 37868 articles, 286 papers were shortlisted for further review. From these, 17 qualitative studies describing the factors influencing obesogenic dietary intake in young children (0-6 years) were included in the review. Three additional papers were identified in August 2013, two ${ }^{35} 36$ through correspondence with first authors of included studies and one ${ }^{37}$ from the personal database of one of the authors (RL).

All excluded studies were recorded with details and reasons for exclusion. Nineteen papers, mainly conference abstracts or PhD theses, without peer-reviewed fulltext papers, were also excluded and have been accounted for in the flow chart (figure 1) as excluded for 'other reasons'.

\section{Data extraction and analyses}

The included qualitative studies $(n=20)$ reported outcomes of interviews, focus groups and participant observation.

A pre-piloted Excel spreadsheet was used to collect information about the study objectives, settings, sample size and selection and other details of the research methods. The data extraction and analysis followed the thematic framework approach underpinned by the socioecological model and included familiarisation of the data across the studies, creating a thematic framework, indexing, charting, mapping and interpretation. ${ }^{38} 39$ Data extraction involved an iterative process of reading and rereading of the studies, the identification of themes concerning views on factors influencing obesogenic dietary intake. A stepwise approach was used to compare and consolidate the initial themes into one list and finally a summary into overarching themes. Details of individual studies (including methods, emergent themes, participant quotes and author conclusions) are documented in online supplementary table S3. The thematic framework was created by RL and double-checked by VMP to ensure agreement and consistency in data extraction and reporting. Any disagreement was resolved by consensus.

\section{RESULTS}

\section{Study characteristics}

The included studies $(n=20)$ had a total of 1067 participants (901 parents/caregivers, 37 children, 87 teachers, 15 dieticians and 27 nursery staff, ie, 9 managers, 6 cooks and 12 other staff). Studies were mainly conducted in developed countries (USA $n=8,{ }^{40-47}$ Mexico $\mathrm{n}=1,{ }^{48}$ Canada $\mathrm{n}=1,{ }^{49}$ Australia $\mathrm{n}=4,{ }^{37}{ }^{50-52}$ UK $\mathrm{n}=3,{ }^{36} 5354$ Sweden $\mathrm{n}=1,{ }^{55}$ multiple $(6$ and 8 countries, respectively) and countries in Europe $n=2,3556$ between 2004 and 2013. Eighteen studies were of high quality and two were of intermediate quality. ${ }^{43}$ Qualitative evidence on SSB intake was identified from 8

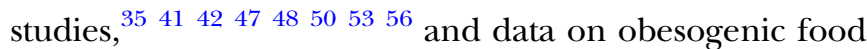

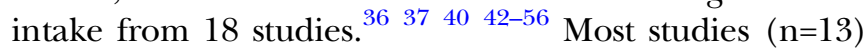


were in non-generalisable populations such as obese children or low-income families, and these were categorised as non-representative of the general population. ${ }^{35-37} 40-475053$

Eleven of the 20 studies aimed to recruit parents, ${ }^{36} 37404146484951535556$ three recruited only mothers, ${ }^{42} 4752$ one had only fathers, ${ }^{43}$ one had only child participants ${ }^{50}$ and four had mixed populations, that is, parents and teachers ${ }^{35}$; mothers and dieticians ${ }^{45}$; mothers and children, ${ }^{44}$ and nursery staff, parents and children. ${ }^{54}$ Overall, the number of fathers recruited per study was small. ${ }^{40} 41 \quad 43 \quad 4648 \quad 55 \quad 56$ The study designs varied: 16 studies held focus groups, 6 interviewed individual participants and 1 was an ethnographic study, ${ }^{54}$ with some studies using more than one data collection method. The number of participants in each study ranged from 6 to 209. Most studies included children aged 2-6 years and only four studies specified that children below 2 years were included. ${ }^{36} 434547$

\section{Thematic analysis}

Several behavioural factors were identified as barriers to or facilitators of healthy diet and, despite of differences in study context and focus, 15 themes were identified with 12 themes reported consistently by three or more studies (see online supplementary table S3). The factors were thematically coded and analysed at three levels as per the socioecological model (table 1): child (1 theme), parental/family (7 themes) and environmental (4 themes). Participant (parent, mother, father, child, teacher, etc) quotes are presented in parentheses to illustrate points in respective themes.

\section{Themes identified}

Child factors

\section{Child's preference/likes}

This was the only child-level theme identified and was reported by 14 studies. These studies found child's taste preferences, attitudes, likes and dislikes towards food/ beverage consumption and difficult feeding/fussy eating to be factors influencing diet in young children, with parents usually offering what the child preferred. ("Your child can influence how you feed them, definitely, depending on their likes and dislikes"-Parent. ${ }^{37}$ "We feel like we've got to give our kids things all the time... because they want it, we have got to take them to McDonalds"-Mother. ${ }^{52}$ ) Caregivers also described strategies to overcome barriers to healthy eating: motivating and encouraging children to make healthy choices, through negotiation, repeated exposure to healthy food and opportunities for children to prepare meals.

\section{Parental/family/peer factors \\ Modelling}

Fifteen studies reported parental/family/peer influences, of which 10 studies reported that negative parental modelling, conflicting food purchases, and parental preference for takeaways and packaged food were barriers to healthy eating in children. ("I've learned that children do copy us...when your husband is having chocolate it's not fair to expect your child to have banana or fruit"-Mother. ${ }^{24}$ ) Fourteen studies reported that family member/s and peers influenced child's dietary behaviours, sometimes challenging parental practices. Older siblings and wider family members were often perceived as role models. ("As a parent, you are responsible for your children's lifestyle. We have to guide our children...Children don't do what parents tell them to; they do what parents do"-Parent. ${ }^{55}$ )

\section{Knowledge, perceptions, beliefs, confidence, motivation and cooking skills}

Fourteen studies reported that such parental influences affect their child's diet. Many parents reported poor nutritional knowledge/education, were more concerned about child's underweight status than overweight, or

Table 1 Summary of factors influencing young children's obesogenic diet

\section{Broad themes}

\section{A. Child factors:}

1. Preference/likes

B. Parental factors:

2. Modelling (parental/ family/peer)

3. Knowledge, perceptions, beliefs, confidence, motivation and cooking skills

4. Time and convenience

5. Using food as a reward

6. Affordability/cost

7. Concerns about child's health

8. Involving children in food preparation

C. Environmental factors:

9. Societal/cultural influence

10. Preschool/childcare/healthcare influence

11. Availability of obesogenic food and drinks

12. Advertising and packaging

\section{Studies* exploring each theme}

$2,3,4,5,7,8,9,10,13,14,16,17,19,20$

$1,2,3,4,7,9,10,11,12,13,14,17,18,19,20$

$1,2,6,7,8,10,11,13,14,15,16,17,18,19$

$2,3,4,7,10,11,14,15,17,18$

$3,5,7,11,12,13,15,16,17,19$

$2,3,4,10,11,13,14,17,18$

$3,5,7,9,10,13,14,15,18$

$12,13,17$

$3,4,11,15,17$

$1,3,4,6,7,9,10,11,13,14$

$1,4,5,7,8,10,11,12,15,17$

$1,2,3,4,7,9,10,11,12,13$

*Study number as per online supplementary table S3. 
were overweight themselves, which limited accurate identification of their child's weight status. Some studies reported unhealthy food preparation and feeding practices, early weaning and early introduction of solids as factors affecting healthy diet in infants and toddlers. ("They talk to you and educate you about nutrition, they teach you...I did not know about the pyramid of food, the amount of bread, the amount of rice and fruit...and that is a big influence"-Mother. ${ }^{42}$ ) One study reported that lack of support, confidence, motivation and cooking skills was a barrier to healthy eating in children. ("The confidence I think could be (a barrier to providing healthy food), yeah, thinking, oh my goodness I'm going to mess that meal up, I'm going to go for the easy option"-Parent. ${ }^{36}$ )

\section{Time and convenience}

Ten studies reported influences on this theme. Parents resorted to convenience food to help them juggle jobs and children's activities, and lack of time clearly influenced parents' choices and affected children's diets. ("It's hard, so you almost give up good eating for activities...so they either eat good and don't get a good activity, or you get a good activity and you eat at McDonald's"-Parent. ${ }^{49}$ "I don't cook and I'm the only one who can cook...but I am never there. There will be nights I'll work 18 hours straight and I'll be up at 6:00... and go to work... When I do feed her, it's McDonald's, Burger King, something like that. I can grab and go... because I don't have the time"-Caregiver. ${ }^{40}$ )

\section{Using food as a reward}

Ten studies reported that parents used obesogenic food as a reward for good behaviour. ("She then asked for a chocolate from the Christmas tree. I said she could have one if she ate a Satsuma first, which she did." "Because she had eaten all of her food, I said, do you want some ice-cream?"-Parent. ${ }^{53}$ ) This practice could directly increase obesogenic food intake and also make it more desirable for children.

\section{Affordability/cost}

Nine studies reported the influence of financial constraints. Most of these studies reported that participants felt they could not afford to purchase healthy food for their children, which they perceived to be more expensive than unhealthy food. ("A limited household income should not be the same as living an unhealthy life. The government should subsidise healthy...food." "Healthier food should cost less. I cannot believe that fruit and vegetables should be so terribly expensive"-Parent. ${ }^{55}$ )

\section{Concerns about child's health}

Nine studies reported that parents' or carers' concerns about their child's health influenced their dietary decisions, usually positively but also in some cases adversely. (“... and during some of that time I'll let him eat that without arguing about eating the junk food a lot of times just as long as I know he's filling up on something"-Parent. ${ }^{40}$ )

\section{Involving children in food preparation}

Three studies reported that involving children in food preparation, being creative with food, making mealtime special (eg, picnic), mealtime rituals and routines, could facilitate healthy eating. ("I try to get them into the kitchen as much as I can, especially if I know that they don't like the dish that I am preparing"-Parent. ${ }^{51}$ )

\section{Environmental factors \\ Societal/cultural influence}

Five studies reported that societal and cultural pressures influenced children's dietary intake while some parents found it difficult to manage without social support. ("Well, I think society has a responsibility for our children's lifestyle, since the politicians make the laws. I believe in early prevention..."-Parent. ${ }^{55}$ ) Social norms and cultural challenges such as influence of friends and social occasions, social support from grandparents and friends, and SSB consumption at home being a norm in some cultures adversely influenced dietary intakes. ("In our culture, children are allowed to have sweets several times a week...it's not a big deal"-Parent. ${ }^{55}$ )

\section{Preschool/childcare/healthcare influence}

Ten studies reported that preschool/childcare and healthcare policies influenced children's dietary intake. Parents and teachers acknowledged that both were role models with collective responsibility towards children and that childcare settings should provide and assist parents to provide healthy food to children. ("When children have chocolate milk or other sugared sweetened beverages at school every day, the preschool will have a stimulating and key role for the intake of sugar"-Parent. ${ }^{35}$ "We cannot control what they eat and their diet really depends on the place where they stay during the day"-Parent. ${ }^{56}$ )

Parents expected preschool to also provide them with information on healthy eating, so parents could promote healthy dietary intake within the family unit. ("Preschool affects our children's lifestyle. Children spend all day there...preschools should have the mission to coach parents to raise children with healthy lifestyles...parents groups for instance..."-Parent. ${ }^{55}$ )

\section{Availability of obesogenic food and drinks}

Ten studies reported that children tend to consume obesogenic food and drink whenever it was available, at home or at school. ("Probably the most obvious thing (influencing a child's food preferences) to me is what is available. If we have certain foods in the house she will eat them, if we don't, she doesn't ask for them"Parent. ${ }^{51}$ "My children only drink cartons of orange juice. I have nothing else"-Parent. ${ }^{35}$ ) Controlling of home and school food environment and limiting the availability/accessibility of unhealthy food was 
considered to facilitate a healthy diet in children. ("I keep an eye on what she actually is eating because I'm sure if I put a big tin of Quality Street out in front of her, she'd quite happy demolish as many as possible"Parent. ${ }^{53}$ )

\section{Advertising and packaging}

Ten studies reported that children and parents are influenced by marketing of obesogenic foods. Advertising, food packaging, food placement in supermarkets, and high availability of unhealthy foods in public places made it difficult for parents to make healthy food choices. ("I think when sweets are obviously in the shops and advertising them and the children can see them, it makes it very hard obviously to get away from that with them demanding things like that"-Parent. ${ }^{36}$ "Children are exposed to temptation all of the time, both in the shops, on television and in newspapers"-Parent. ${ }^{55}$ )

\section{DISCUSSION}

Previous systematic reviews have stated that there is lack of evidence on the factors influencing obesogenic dietary intake in young children. ${ }^{10} 1314$ Our systematic review of qualitative evidence identified many behavioural (parent/carer and child) and environmental (home/preschool/childcare and wider environment) factors, reported by parents and carers as influencing obesogenic dietary intake in young preschool-aged children. Almost all of the studies (18/20) were of 'high' methodological quality. Ten of the 12 factors/themes (per socioecological model) were reported by nine or more studies and 2/12 were reported by three and five studies, respectively.

At the child's level; child's dietary preferences/likes were consistently reported by 14 studies as a major factor in child's obesogenic dietary intake. These could be appetite-related behaviours which may be enhanced by environmental influences. ${ }^{57}$ Also, preschool-aged children potentially make inappropriate food choices in the absence of parental supervision. ${ }^{58}$ Child preferences also influenced parental feeding decisions, so promoting an authoritative parenting style in addition to teaching parents easy to cook, tasty, healthy and affordable foods appears to be important.

At the parental/family level, negative parent/family/ peer modelling, lack of knowledge, time constraints, use of food as a reward, affordability and concerns about child's health were studied extensively by more than eight studies each and were identified as influencers of children's obesogenic dietary intake. These findings are consistent with other research on the topic. ${ }^{10} 1359$ Parental, family and peer modelling was reported extensively by 15 studies and strongly influences the child's obesogenic dietary intake. Hence, promoting parental positive modelling should be included in any intervention. Quantitative evidence has shown that early weaning from exclusive breastfeeding ${ }^{60}$ and introduction to non-core foods early ${ }^{61}{ }^{62}$ was significantly associated with increased obesogenic dietary intake in young children. Studies have shown that dietary intake of children is dependent on who feeds the child. ${ }^{63}$ We found that choice of obesogenic dietary intake in children had influences from social, environmental and behavioural domains. Recent qualitative studies have also reported similar findings. ${ }^{35}{ }^{64-69}$ Preschool-aged children rely on their parents or carers for food and some children may be more vulnerable to parental unhealthy feeding habits and strategies.

Maternal factors were studied more extensively than those of paternal or other carers; perhaps this is because mothers spend more time with their preschoolers and it is easier to recruit mothers to studies. Also, evidence suggests that maternal factors are associated with increased obesity in children. ${ }^{70} 71$ Three studies in our review reported that children who were involved in the food preparation ate healthy meals. This was also the conclusion of a recent qualitative study. ${ }^{72}$ We also found that parents who recognised childhood obesity as a problem tried to promote healthy eating habits in their children. So improving parent's identification and understanding of obesity may be a useful intervention strategy.

At the environmental level; availability of obesogenic food/drinks, advertising, societal, cultural and preschool/childcare influences were identified as factors influencing obesogenic dietary intake in children. Quantitative evidence shows a positive association with availability and obesogenic dietary behaviours ${ }^{73}$ and that home food availability mediates the association between maternal nutrition knowledge and child's diet. ${ }^{74} \mathrm{We}$ found that parents could make changes to the home food environment to promote a healthy diet. Our review also found that parents expect day care/preschool providers, paediatricians and policymakers to help improve their nutritional knowledge and the child's dietary intake. Perhaps capacity building and training of staff at nursery/preschool, and developing interventions integrating social class and culture into ecological frameworks, may be effective in obesity prevention through the identification and development of culturally sensitive nutritional information. ${ }^{75}{ }^{76}$ Environmental factors such as supermarkets/convenience stores (food placement, offers), food pricing, food availability and food advertising influence the child's diet. It would be useful to investigate whether changing environmental determinants such as advertising, marketing, packaging, pricing and food positioning could reduce obesogenic dietary intake in young children. ${ }^{77} 78$

The collective findings of our review concur with the larger body of evidence in older children and adolescents that knowledge, taste preferences, school policies, parental modelling, influence of peers, availability and accessibility all influence eating behaviours. ${ }^{20}{ }^{79-82}$ Our findings are also similar to those of a recent Australian qualitative study and a systematic review which investigated only parental influences on dietary intake in 
children aged 2-5 years and highlighted the following factors: cultural differences, parental attitudes, perceptions and concerns; barriers faced by lower income families to providing healthy foods; using food to shape child's behaviour; lack of understanding of weight status and obesity consequences; inappropriate parent feeding practices and nutrition knowledge. ${ }^{32} 72$

\section{STRENGTHS AND WEAKNESSES OF THE REVIEW}

To the best of our knowledge, this is the first systematic review of qualitative evidence on the factors influencing obesogenic dietary intake in preschool-aged children. We conducted a comprehensive search without period or language restrictions, as well as handsearched and contacted authors of included studies to identify relevant literature. We also followed preset inclusion criteria and systematic review procedures throughout to minimise selection and reviewer-related biases. All but 2 of the 20 included studies were assessed to be of 'high' methodological quality and only 2 were of 'intermediate' quality. Ten of the 12 factors/themes were consistently reported by at least nine studies. Seventeen of the 20 studies were published after 2003, indicating that most research on this topic is contemporary. Relevant studies up to June 2014 have been included in our review and this may be a limitation, but we believe that recent qualitative studies will not significantly alter our conclusions. Studies included in this review were almost all from developed countries, although a few were set in the UK. Furthermore, very few fathers or carers were included as participants, which limits the scope of the findings.

\section{CONCLUSIONS}

There is consistent qualitative evidence that several factors at various levels of the socio-ecological model (child, parent and environment), influence obesogenic dietary intake, although modelling appears to have the most influence. These findings support the rationale for the design and testing of multilevel interventions to reduce obesogenic dietary intake in young preschool age children. Such interventions should also target the barriers to healthy eating-affordability, convenience, modelling, education, child preference, availability and advertising.

Acknowledgements The authors sincerely thank the following collaborators on the project: Dr Helen Moore for performing the search in electronic databases; Mrs Claire O'Malley and Dr Kathryn Hesketh for helping with the screening of titles and abstracts; Dr Esther van Sluijis, Professor Carolyn Summerbell and Professor Simon Griffin for their comments and guidance through the project. They also thank the following from the University of Cambridge: Mrs Sheila Palmer and Miss Isla Kuhn for their help with literature sourcing; Professor Carol Brayne, Dr Louise Lafortune, Ms Francis Cater and Ms Judith Willows for their help and support throughout.

Contributors VMP was the lead reviewer with $\mathrm{RL}$ as the paired senior reviewer and overall obesity project lead. KKO reviewed and edited the manuscript. All the authors contributed to the study design, critical revision of the manuscript and approved the final version.
Funding This independent research is funded by the National Institute of Health Research, School for Public Health Research (NIHR SPHR). The National Institute for Health Research's School for Public Health Research (NIHR SPHR) is a partnership between the Universities of Sheffield, Bristol, Cambridge, UCL; The London School for Hygiene and Tropical Medicine; The Peninsula College of Medicine and Dentistry; the LiLaC collaboration between the Universities of Liverpool and Lancaster and Fuse; The Centre for Translational Research in Public Health, a collaboration between Newcastle, Durham, Northumbria, Sunderland and Teesside Universities. The work was undertaken under the auspices of the Centre for Diet and Activity Research (CEDAR), a UKCRC Public Health Research Centre of Excellence which is funded by the British Heart Foundation, Cancer Research UK, Economic and Social Research Council, Medical Research Council, the National Institute for Health Research, and the Wellcome Trust (RES-590-28-0002).

\section{Competing interests None declared.}

Provenance and peer review Not commissioned; externally peer reviewed.

Data sharing statement No additional data are available.

Open Access This is an Open Access article distributed in accordance with the Creative Commons Attribution Non Commercial (CC BY-NC 4.0) license, which permits others to distribute, remix, adapt, build upon this work noncommercially, and license their derivative works on different terms, provided the original work is properly cited and the use is non-commercial. See: http:// creativecommons.org/licenses/by-nc/4.0/

\section{REFERENCES}

1. Ogden CL, Carroll MD, Kit BK, et al. Prevalence of obesity and trends in body mass index among US children and adolescents, 1999-2010. JAMA 2012;307:483-90.

2. de Onis M, Blössner M, Borghi E. Global prevalence and trends of overweight and obesity among preschool children. Am J Clin Nutr 2010;92:1257-64.

3. The Health and Social Care Information Centre LS. National Child Measurement Programme: England, 2012/13 school year; December 2013. 2013. http://www.hscic.gov.uk/catalogue/ PUB13115/nati-chil-meas-prog-eng-2012-2013-rep.pdf

4. Cunningham SA, Kramer MR, Narayan KMV. Incidence of childhood obesity in the United States. N Engl J Med 2014;370:403-11.

5. WHO. Diet, nutrition and the prevention of chronic diseases; Report of a joint WHO/FAO expert consultation. Geneva; World Health Organization, 2003.

6. Lobstein T, Baur L, Uauy R. Obesity in children and young people: a crisis in public health. Obes Rev 2004;5(Suppl 1):4-104.

7. Waters E, de Silva-Sanigorski A, Hall BJ, et al. Interventions for preventing obesity in children. Cochrane Database Syst Rev 2011; (12):CD001871.

8. Lakshman R, Elks CE, Ong KK. Childhood obesity. Circulation 2012;126:1770-9.

9. Li B, Adab P, Cheng KK. Family and neighborhood correlates of overweight and obesogenic behaviors among Chinese children. Int $J$ Behav Med 2014;21:700-9.

10. De Craemer M, De Decker E, De Bourdeaudhuij I, et al. Correlates of energy balance-related behaviours in preschool children: a systematic review. Obes Rev 2012;13:13-28.

11. De Craemer M, Lateva $M$, lotova $\mathrm{V}$, et al. Differences in energy balance-related behaviours in European preschool children: the ToyBox-study. PLOS ONE 2015;10:e0118303.

12. Jones RA, Hinkley $T$, Okely AD, et al. Tracking physical activity and sedentary behavior in childhood: a systematic review. Am J Prev Med 2013;44:651-8.

13. te Velde SJ, van Nassau F, Uijtdewilligen L, et al. Energy balance-related behaviours associated with overweight and obesity in preschool children: a systematic review of prospective studies. Obes Rev 2012;13:56-74.

14. Hesketh KD, Campbell KJ. Interventions to prevent obesity in 05-year-olds: an updated systematic review of the literature. Obesity (Silver Spring) 2010;18(Suppl 1):S27-35.

15. te Velde SJ, Singh A, Chinapaw M, et al. Energy balance related behaviour: personal, home- and friend-related factors among schoolchildren in Europe studied in the ENERGY-project. PLoS ONE 2014;9:e111775.

16. Kremers SP, de Bruijn GJ, Visscher TL, et al. Environmental influences on energy balance-related behaviors: a dual-process view. Int J Behav Nutr Phys Act 2006;3:9. 
17. DeBoer MD, Scharf RJ, Demmer RT. Sugar-sweetened beverages and weight gain in 2- to 5-year-old children. Pediatrics 2013;132:413-20.

18. Papandreou D, Andreou E, Heraclides A, et al. Is beverage intake related to overweight and obesity in school children? Hippokratia 2013;17:42-6.

19. Mathias KC, Slining MM, Popkin BM. Foods and beverages associated with higher intake of sugar-sweetened beverages. Am J Prev Med 2013;44:351-7.

20. Shepherd J, Harden A, Rees R, et al. Young people and healthy eating: a systematic review of research on barriers and facilitators. Health Educ Res 2006;21:239-57.

21. Reilly JJ, Kelly J. Long-term impact of overweight and obesity in childhood and adolescence on morbidity and premature mortality in adulthood: systematic review. Int J Obes (Lond) 2011;35:891-8.

22. Han JC, Lawlor DA, Kimm SY. Childhood obesity. Lancet 2010;375:1737-48

23. Ambrosini GL. Childhood dietary patterns and later obesity: a review of the evidence. Proc Nutr Soc 2014;73:137-46.

24. WHO. World Health Organization: Report of the first meeting of the ad hoc working group on science and evidence for ending childhood obesity; 2014. Geneva, Switzerland, 18-20 June 2014

25. Kelder SH, Perry CL, Klepp KI, et al. Longitudinal tracking of adolescent smoking, physical activity, and food choice behaviors. Am J Public Health 1994;84:1121-6.

26. Northstone K, Emmett PM. Are dietary patterns stable throughout early and mid-childhood? A birth cohort study. Br J Nutr 2008;100:1069-76.

27. Mikkila V, Rasanen L, Raitakari OT, et al. Consistent dietary patterns identified from childhood to adulthood: the cardiovascular risk in Young Finns Study. Br J Nutr 2005;93:923-31.

28. Marshall TA. Preventing dental caries associated with sugar-sweetened beverages. J Am Dent Assoc 2013;144:1148-52.

29. Catteau C, Trentesaux T, Delfosse $C$, et al. [Consumption of fruit juices and fruit drinks: impact on the health of children and teenagers, the dentist's point of view]. Arch Pediatr 2012;19:118-24.

30. Martin J, Chater A, Lorencatto F. Effective behaviour change techniques in the prevention and management of childhood obesity. Int J Obes 2013;37:1287-94.

31. Nixon CA, Moore HJ, Douthwaite W, et al. Identifying effective behavioural models and behaviour change strategies underpinning preschool- and school-based obesity prevention interventions aimed at 4-6-year-olds: a systematic review. Obes Rev 2012;13:106-17.

32. Peters J, Parletta N, Campbell K, et al. Parental influences on the diets of 2- to 5-year-old children: systematic review of qualitative research. J Early Child Res 2014;12:3-19.

33. Lakshman R, Mazarello Paes V, Hesketh K, et al. Protocol for systematic reviews of determinants/correlates of obesity-related dietary and physical activity behaviors in young children (preschool 0 to 6 years): evidence mapping and syntheses. Syst Rev 2013;2:28.

34. Evidence for Policy and Practice Information (EPPI) and Co-ordinating Centre loE, University of London. EPPI-Centre Methods for Conducting Systematic Reviews. March 2007, Updated 2010. http://eppi.ioe.ac.uk/cms/LinkClick.aspx? fileticket=hQBu8y4uVwl\%3d\&tabid=1919\& mid $=6163$

35. De Craemer M, De Decker E, De Bourdeaudhuij I, et al. Physical activity and beverage consumption in preschoolers: focus groups with parents and teachers. BMC Public Health 2013;13:278.

36. Hayter AKM, Draper AK, Ohly HR, et al. A qualitative study exploring parental accounts of feeding pre-school children in two low-income populations in the UK. Matern Child Nutr 2015;11:371-84.

37. Petrunoff NA, Wilkenfeld RL, King LA, et al. 'Treats', 'sometimes foods', 'junk': a qualitative study exploring 'extra foods' with parents of young children. Public Health Nutr 2014;17:979-86.

38. Thomas J, Harden A. Methods for the thematic synthesis of qualitative research in systematic reviews. BMC Med Res Methodol 2008;8:45.

39. Gale N, Heath $\mathrm{G}$, Cameron $\mathrm{E}$, et al. Using the framework method for the analysis of qualitative data in multi-disciplinary health research. BMC Med Res Methodol 2013;13:117.

40. Omar MA, Coleman G, Hoerr S. Healthy eating for rural low-income toddlers: caregivers' perceptions. J Community Health Nurs 2001;18:93-106.

41. Bolling C, Crosby L, Boles R, et al. How pediatricians can improve diet and activity for overweight preschoolers: a qualitative study of parental attitudes. Acad Pediatr 2009:9:172-8.

42. Lindsay AC, Sussner KM, Greaney ML, et al. Influence of social context on eating, physical activity, and sedentary behaviors of Latina mothers and their preschool-age children. Health Educ Behav 2009;36:81-96.
43. A Horodynski M, Arndt MJ. "Eating-together" mealtimes with African-American fathers and their toddlers. Appl Nurs Res 2005; 18:106-9.

44. Sherry B, McDivitt J, Birch LL, et al. Attitudes, practices, and concerns about child feeding and child weight status among socioeconomically diverse white, Hispanic, and African-American mothers. J Am Diet Assoc 2004;104:215-21.

45. Baughcum AE, Burklow KA, Deeks CM, et al. Maternal feeding practices and childhood obesity: a focus group study of low-income mothers. Arch Pediatr Adolesc Med 1998;152:1010-14.

46. Styles JL, Meier A, Sutherland LA, et al. Parents' and caregivers' concerns about obesity in young children: a qualitative study. Fam Community Health 2007;30:279-95.

47. Auld GW, Morris M. Infant/toddler feeding practices of adolescent mothers: ethnic differences and intergenerational involvement. Fam Cons Sci Res J 1994;23:118-34.

48. Rodriguez-Oliveros G, Haines J, Ortega-Altamirano D, et al. Obesity determinants in Mexican preschool children: parental perceptions and practices related to feeding and physical activity. Arch Med Res 2011;42:532-9.

49. Tucker P, Irwin JD, He M, et al. Preschoolers' dietary behaviours: parents' perspectives. Can J Diet Pract Res 2006;67:67-71.

50. Fitzgerald E, Bunde-Birouste A, Webster E. Through the eyes of children: engaging primary school-aged children in creating supportive school environments for physical activity and nutrition. Health Promot J Aust 2009;20:127-32.

51. Campbell KJ, Crawford DA, Hesketh KD. Australian parents' views on their 5-6-year-old children's food choices. Health Promot Int 2007:22:11-18.

52. Pagnini DL, Wilkenfeld RL, King LA, et al. Mothers of pre-school children talk about childhood overweight and obesity: the weight of opinion study. J Paediatr Child Health 2007;43:806-10.

53. Carnell S, Cooke L, Cheng R, et al. Parental feeding behaviours and motivations. A qualitative study in mothers of UK pre-schoolers. Appetite 2011;57:665-73.

54. Lloyd-Williams F, Bristow K, Capewell S, et al. Young children's food in Liverpool day-care settings: a qualitative study of pre-school nutrition policy and practice. Public Health Nutr 2011;14:1858-66.

55. Stenhammar C, Wells M, Ahman A, et al. 'Children are exposed to temptation all the time'-parents' lifestyle-related discussions in focus groups. Acta Paediatr 2012;101:208-15

56. Haerens L, De Bourdeaudhuij I, Barba G, et al. Developing the IDEFICS community-based intervention program to enhance eating behaviors in 2- to 8-year-old children: findings from focus groups with children and parents. Health Educ Res 2009;24: 381-93.

57. Dubois L, Diasparra M, Bedard B, et al. Genetic and environmental influences on eating behaviors in 2.5- and 9-year-old children: a Iongitudinal twin study. Int J Behav Nutr Phys Act 2013;10:134.

58. Briefel RR, Crepinsek MK, Cabili C, et al. School food environments and practices affect dietary behaviors of US public school children. $J$ Am Diet Assoc 2009;109:S91-107.

59. Maliwichi L, Nesengani A. An investigation into child feeding practices of young mothers from Tshilata village, Vuwani, Limpopo province, South Africa. Ann Nutr Metab 2013;63:543.

60. Brekke HK, van Odijk J, Ludvigsson J. Predictors and dietary consequences of frequent intake of high-sugar, low-nutrient foods in 1 -year-old children participating in the ABIS study. $\mathrm{Br} J$ Nutr 2007;97:176-81.

61. Koh GA, Scott JA, Oddy WH, et al. Exposure to non-core foods and beverages in the first year of life: results from a cohort study. Nutr Diet 2010;67:137-42.

62. Campbell KJ, Lioret S, McNaughton SA, et al. A parent-focused intervention to reduce infant obesity risk behaviors: a randomized trial. Pediatrics 2013;131:652-60.

63. Wasser HM, Thompson AL, Maria Siega-Riz A, et al. Who's feeding baby? Non-maternal involvement in feeding and its association with dietary intakes among infants and toddlers. Appetite 2013;71:7-15.

64. Beck AL, Takayama JI, Halpern-Felsher B, et al. Understanding how Latino parents choose beverages to serve to infants and toddlers. Matern Child Health J 2014;18:1308-15.

65. Hoare A, Virgo-Milton M, Boak R, et al. A qualitative study of the factors that influence mothers when choosing drinks for their young children. BMC Res Notes 2014;7:430.

66. Goto K, Ominami C, Song CY, et al. Globalization, localization and food culture: perceived roles of social and cultural capitals in healthy child feeding practices in Japan. Glob Health Promot 2014;21:50-8.

67. Lindsay A, Greaney M, Sussner K, et al. Socio-cultural, organizational, and community level influences on physical activity levels of Latino preschool-aged children: a qualitative study. Ann Nutr Metab 2013;63:432. 
68. Duncanson K, Burrows T, Holman B, et al. Parents' perceptions of child feeding: a qualitative study based on the theory of planned behavior. J Dev Behav Pediatr 2013;34:227-36.

69. Herman AN, Malhotra K, Wright G, et al. A qualitative study of the aspirations and challenges of low-income mothers in feeding their preschool-aged children. Int J Behav Nutr Phys Act 2012;9:132.

70. Van Den Berg G, Van Eijsden M, Galindo-Garre F, et al. Low maternal education is associated with increased growth velocity in the first year of life and in early childhood: the ABCD study. Eur $J$ Pediatr 2013;172:1451-7.

71. Lakshman R, Zhang J, Zhang J, et al. Higher maternal education is associated with favourable growth of young children in different countries. J Epidemiol Community Health 2013;67:595-602.

72. Peters J, Parletta N, Lynch J, et al. A comparison of parental views of their pre-school children's 'healthy' versus 'unhealthy' diets. A qualitative study. Appetite 2014;76:129-36.

73. Bogart LM, Cowgill BO, Sharma AJ, et al. Parental and home environmental facilitators of sugar-sweetened beverage consumption among overweight and obese Latino youth. Acad Pediatr 2013;13:348-55.

74. Campbell KJ, Abbott G, Spence AC, et al. Home food availability mediates associations between mothers' nutrition knowledge and child diet. Appetite 2013;71:1-6.

75. Mena NZ, Gorman K, Dickin K, et al. Contextual and Cultural Influences on Parental Feeding Practices and Involvement in Child
Care Centers among Hispanic Parents. Childhood obesity 2015;11:347-54.

76. Mohammadpour-Ahranjani B, Pallan MJ, Rashidi A, et al. Contributors to childhood obesity in Iran: the views of parents and school staff. Public Health 2014;128:83-90.

77. Horsley JA, Absalom KA, Akiens EM, et al. The proportion of unhealthy foodstuffs children are exposed to at the checkout of convenience supermarkets. Public Health Nutr 2014;17:2453-8.

78. Jenkin G, Madhvani N, Signal L, et al. A systematic review of persuasive marketing techniques to promote food to children on television. Obes Rev 2014;15:281-93

79. Patrick $\mathrm{H}$, Nicklas TA. A review of family and social determinants of children's eating patterns and diet quality. J Am Coll Nutr 2005;24:83-92.

80. Wind M, Bobelijn K, De Bourdeaudhuij I, et al. A qualitative exploration of determinants of fruit and vegetable intake among 10- and 11-year-old schoolchildren in the low countries. Ann Nutr Metab 2005;49:228-35. doi:10.1159/000087246

81. De Bourdeaudhuij I, Yngve A, te Velde SJ, et al. Personal, social and environmental correlates of vegetable intake in normal weight and overweight 9 to 13-year old boys. Int J Behav Nutr Phys Act 2006;3:37.

82. O'Dea JA. Why do kids eat healthful food? Perceived benefits of and barriers to healthful eating and physical activity among children and adolescents. J Am Diet Assoc 2003;103:497-501. 